\title{
Influência da via de parto sobre os resultados perinatais de mulheres que tiveram parto prematuro
}

\section{The influence of the route of delivery on perinatal outcomes in women who had preterm delivery}

Telma Gurgel Costa Santos Mota ${ }^{1}$. Carlos Augusto Alencar Junior ${ }^{2}$. Gilberto Gomes Ribeiro ${ }^{3}$. Igor Studart de Lucena Feitosa ${ }^{4}$. Francisco Edson de Lucena Feitosa ${ }^{5}$.

1 Residência em Tocoginecologia, Maternidade Escola Assis Chateaubriand (MEAC), Universidade Federal do Ceará (UFC), Fortaleza, Ceará, Brasil. 2 Professor Associado do Departamento de Saúde Materno-Infantil, Universidae Federal do Ceará (UFC), Fortaleza, Ceará, Brasil. 3 Preceptor da Residência em Tocoginecologia, Maternidade Escola Assis Chateaubriand (MEAC), Universidade Federal do Ceará (UFC), Fortaleza, Ceará, Brasil. 4 Acadêmico da Faculdade de Medicina, Universidade Federal do Ceará (UFC), Fortaleza, Ceará, Brasil. 5 Professor Adjunto do Departamento de Saúde Materno-Infantil, Universidade Federal do Ceará (UFC), Fortaleza, Ceará, Brasil.

\section{RESUMO}

Objetivos: avaliar a influência da via de parto sobre os resultados perinatais em mulheres que tiveram parto prematuro; avaliar características demográficas e obstétricas como determinantes da via de parto. Métodos: realizou-se estudo transversal na Maternidade Escola Assis Chateaubriand, com 195 mulheres que tiveram parto prematuro, sem complicações e os recém-nascidos com peso $\geq 500 \mathrm{~g}$ e idade gestacional entre 22 e 36 semanas e seis dias. Para avaliação estatística comparativa entre os dois grupos (partos vaginal e abdominal), utilizou-se o teste de Mann-Whitney. O cálculo da razão de risco ajustado foi realizado através de Regressão Logística e Multivariada. Resultados: observou-se que o pródromo de trabalho de parto prematuro, bolsa rota e administração de tocolíticos e corticoides aumentaram o risco de cesárea, enquanto o trabalho de parto prematuro ativo diminuiu. A cesárea aumentou a chance do Apgar ao $5^{\circ}$ minuto ser $\geq 7$. Conclusões: não foram encontradas diferenças estatisticamente significativas nos resultados perinatais entre recém-nascidos de partos vaginal e abdominal de mulheres que tiveram parto prematuro. O pródromo de trabalho de parto prematuro, bolsa rota e uso de agentes tocolíticos e corticoides aumentaram o risco de cesárea, enquanto o trabalho de parto prematuro ativo diminuiu.

Palavras-chave: Trabalho de parto prematuro. Cesárea. Parto normal. Recém-nascido. Prematuro.

\section{ABSTRACT}

Purpose: to evaluate the influence of route of delivery on perinatal outcomes in women who had preterm delivery; to evaluate demographic and obstetric characteristics as determinants of mode of delivery. Methods: cross-sectional study was conducted in the Maternidade Escola Assis Chateaubriand, with 195 women who had preterm delivery, without complications and newborns weighing $\geq 500 \mathrm{~g}$ and gestational age between 22 and 36 weeks and six days. For the comparative statistical analysis among the two groups (vaginal and abdominal delivery), it was utilized the Mann-Whitney test. The calculation of the reason of adjusted risk was accomplished through Logistical and Multinomial Regression. Results: when the two groups were compared, it was observed that false preterm delivery, premature rupture of the membranes and administration of tocolytics and corticosteroids increased the risk of cesarean section, while the active premature labor decreased. The cesarean section increased the chance of the Apgar score at $5^{\circ}$ minute to be $\geq 7$. Conclusions: it had not been found statistically significant differences in perinatal outcomes among newborns of vaginal delivery and cesarean section in women who had preterm delivery. False preterm delivery, premature rupture of the membranes and the use of tocolytic agents and corticosteroids increased the risk of cesarean section, while the active preterm delivery decreased.

Keywords: Obstetric labor premature. Cesarean section. Natural childbirth. Infant newborn. Infant premature.

Autor correspondente: Telma Gurgel Costa Santos Mota, Rua Dom Expedito Lopes, 2250, apartamento 701, bloco B, Dionísio Torres, Fortaleza, Ceará. CEP: 60135-410.Telefone: +55 85 99958-0274. E-mail: telmagmota@hotmail.com

Conflito de interesses: Não há qualquer conflito de interesses por parte de qualquer um dos autores.

Recebido em: 30 Jan 2017; Revisado em: 21 Mar 2017; Aceito em: 21 Mar 2017. 


\section{INTRODUÇÃO}

Em 1995 o American College of Obstetricians and Gynecologists (ACOG) definiu parto prematuro (PP) como todo aquele que ocorre antes de completadas 37 semanas de gestação, contadas a partir do primeiro dia da última menstruação, independente do peso do recém-nascido (RN). O limite, que é usado para distinguir PP de aborto espontâneo, varia de acordo com o local. ${ }^{1}$ No mundo inteiro a taxa estimada de partos prematuros é cerca de $11 \%{ }^{2}$

O PPé multifatorial e classificado em duas categorias: por indicação médica, que ocorre em $30 \%$ dos casos e espontâneo(com membranas intactas, responsável por $45 \%$ dos mesmos e rotura prematura de membranas (RPM) correspondendo aos demais $25 \%$ ). ${ }^{1,2}$

Seu diagnóstico é feito quando contrações uterinas persistentes são acompanhadas de dilatação e/ou apagamento do colo uterino. ${ }^{3}$ Quando há indicação de inibição, uma variedade de drogas tocolíticas têm sido usadas, porém nenhuma delas é completamente efetiva. ${ }^{4}$

Múltiplos trabalhos têm demonstrado que o uso de glicocorticoides naquelas pacientes com risco para PP diminui a mortalidade neonatal e morbidades como: síndrome do desconforto respiratório (SDR), hemorragia intraventricular (HIV), enterocolite necrotizante (ECN), persistência do canal arterial (PCA) e displasia bronco-pulmonar. ${ }^{5,6}$

O Royal College of Obstetriciaans and Gynaecologists publicou um guideline sobre o uso de corticoides pré-natais na redução da morbimortalidade neonatal, orientando as seguintes condutas com níveis de evidência $\mathrm{A}:^{7}$

- um único curso de corticoide está associado a diminuição significativa das taxas de mortalidade neonatal, SDR e HIV;

- gestações em risco de PP entre 24 e 34 semanas e 06 dias devem fazer uso do corticoide. Em gestações entre 23 semanas e 23 semanas e 06 dias pode ser considerado seu uso, porém, com nível de evidência C. Seu uso está indicado em cesáreas eletivas antes das 38 semanas e 06 dias e nas gestações com restrição de crescimento em risco de PP entre 24 e 35 semanas e 06 dias. Gestações gemelares ou história pregressa de PP que não apresentem risco de PP atual não devem fazer uso;

- a eficácia na diminuição da SDR se dá após 24h até 7 dias depois da administração da segunda dose do corticoide. Entretanto, a diminuição da mortalidade já reduz nas primeiras $24 \mathrm{~h}$ e, portanto, deve ser usada mesmo nas gestações onde a previsão de resolução se dará dentro desse prazo. Estudos melhores precisam ser realizados para esclarecer se essa associação entre o tempo de resolução e o uso do corticoide é real;

- um único curso de corticoide não parece ter qualquer efeito adverso materno ou fetal a curto prazo;

- a posologia deve ser de duas doses de $12 \mathrm{mg}$ de betametasona intramuscular (IM) com intervalo de $24 \mathrm{~h}$ entre as doses ou quatro doses de $6 \mathrm{mg}$ de dexametasona IM a intervalos de $12 \mathrm{~h}$. Doses semanais repetidas não devem ser utilizadas.
Profilaxia para prevenir infecção neonatal pelo estreptococo do grupo B é recomendada para gestantes em trabalho de parto prematuro (TPP), cuja cultura para o mesmo, colhida através de $s w a b$ anal e vaginal é positiva ou então é desconhecida. ${ }^{8}$

Os dados da literatura são unânimes em citar que, concomitantemente com o aumento na incidência de partos prematuros e na sobrevida de recém-nascidos (RNs) pré-termo, nas últimas décadas, tem-se observado também aumento nos índices de cesárea, cujas razões não têm sido completamente investigadas, mas provavelmente têm origem multifatorial, em parte devido ao aumento nas intervenções médicas. ${ }^{9}$ Diversos estudos foram realizados levando em consideração, apresentação fetal, peso fetal, idade gestacional entre outras variantes para determinar as repercussões das vias de parto nos resultados perinatais e maternos, porém, os resultados são controversos e sem significância estatística. ${ }^{10-17}$

Não há consenso sobre a via de parto ideal. Estudar a relação entre a via de parto e os resultados perinatais no prematuro é essencial para prover os serviços de saúde em relação às perspectivas futuras quanto a esta controvérsia.

\section{MÉTODOS}

Realizou-se estudo transversal, visando comparar a influência da via de parto sobre os resultados perinatais em RNs de mulheres que tiveram parto prematuro, como também, características demográficas e obstétricas dessas gestantes, determinantes da via de parto. A pesquisa foi desenvolvida, após aprovação do Comitê de Ética (ofício $n^{0}$ 022/08), no período de 01 de janeiro a 31 de dezembro de 2007, na Maternidade Escola Assis Chateaubriand da Universidade Federal do Ceará (MEAC-UFC), em Fortaleza/CE.

Avaliaram-se os prontuários de gestantes admitidas em pródromo de TPP ou em TPP ativo espontâneo, na Emergência de Obstetrícia da MEAC-UFC que tiveram como desfecho parto vaginal ou cesárea. Após o internamento, as pacientes foram submetidas à seguinte abordagem: (1) repouso em decúbito lateral esquerdo; (2) cuidadosa avaliação do colo uterino através do toque vaginal; (3) confirmação da idade gestacional, medida do colo uterino e avaliação fetal através da ultrassonografia; (4) cvaliação da vitalidade fetal e da atividade uterina através de cardiotocografia; (5) rastreamento clínico e laboratorial de infecções; (6) glicocorticoides entre 24 e 34 semanas de gestação e (7) tocólise com nifedipina quando a mesma foi indicada. Os casos onde ocorreram inibição da atividade uterina, sem progressão do trabalho de parto (TP), não foram incluídos no estudo.

Os critérios de inclusão foram: gestantes admitidas em pródromo de TPP e em TPP ativo espontâneo; idade gestacional $\geq 22$ e $\leq 36$ semanas e seis dias; peso do recém-nascido $\geq$ $500 \mathrm{~g}$ e $\leq 2500 \mathrm{~g}$ e peso $>2500 \mathrm{~g}$ se a idade gestacional foi $<37$ semanas; parto vaginal ou cesárea ocorrido na MEAC-UFC. Os critérios de exclusão foram: gestações múltiplas; gestantes com patologias clínicas ou obstétricas; fetos com malformações, hidropisia ou infecções congênitas; alterações 
da vitalidade e do crescimento fetais diagnosticadas na admissão; patologias placentárias e do cordão umbilical; bolsa rota há mais de 18 horas no momento do parto ou ausência de informações sobre esse tempo; RNs com peso $\leq 2500 \mathrm{~g}$ cuja idade gestacional foi $\geq 37$ semanas; RNs transferidos para outras unidades hospitalares em qualquer momento do internamento; natimorto diagnosticado na admissão.

As variáveis utilizadas para análise descritiva foram divididas em dois grupos: características demográficas e obstétricas (idade materna, paridade, idade gestacional no internamento, diagnóstico da fase do trabalho de parto, uso de tocolítico e corticóide, tipo de apresentação fetal, tipo de parto e indicação do parto abdominal) e desfechos perinatais (idade gestacional ao nascer, peso do recém-nascido, adequação do peso à idade gestacional, escores de Apgar ao $1^{\circ}$ e $5^{\circ}$ minutos, admissão em UTI neonatal e indicação, necessidade de ventilação mecânica e surfactante, presença de tocotraumatismos, outras intercorrências e complicações, tempo de internamento em UTI e total, óbito fetal e neonatal e causa do óbito). Os dados foram coletados, através da utilização de um formulário padronizado, diretamente nos prontuários obtidos no arquivo da MEAC-UFC. Após o preenchimento dos formulários, estes foram revisados e corrigidos pelo pesquisador responsável e posteriormente arquivados em programa estatístico de computador SPSS 10.0 (SPSS Co, Chicago, IL, USA).

Inicialmente, realizou-se análise descritiva da amostragem total, dividindo-se, posteriormente, a população do estudo em dois grupos (partos vaginal e abdominal). As variáveis contínuas foram descritas através da média, desvio padrão e mediana, em cada grupo e na população total, ao passo que as variáveis qualitativas o foram através das frequências absolutas (n), relativas $(\%)$ e incidências $(\%)$. A análise comparativa entre esses 2 grupos foi realizada através da estimativa do risco relativo (RR) para os seguintes desfechos: internamento em UTI neonatal, ventilação mecânica, uso de surfactante, tocotraumatismos e óbito neonatal. Os RNs também foram separados conforme idade gestacional e peso ao nascer, sendo observados os principais parâmetros de complicações neonatais (escores de Apgar inferiores a 7 ao $1^{\circ}$ e $5^{\circ}$ minutos, necessidade de internamento em UTI neonatal, utilização de ventilação mecânica, uso de surfactante, presença de tocotraumatismos e óbitos fetal e neonatal). As variáveis quantitativas foram descritas através de medidas de tendência central, enquanto as variáveis qualitativas, o foram através de tabelas.

A análise comparativa entre os dois grupos foi realizada através do teste não paramétrico de Mann-Whitney para as variáveis expressas em média, mediana e desvio-padrão (dp). Para a comparação da incidência entre os grupos, utilizou-se uma medida de risco, a razão de risco e seu respectivo intervalo de confiança (IC) 95\% visando avaliar a significância estatística (os testes foram considerados estatisticamente significativos quando o $\mathrm{p}$ foi $<0.05)$. Regressão logística multivariada foi utilizada para determinar a influência da via de parto sobre os resultados perinatais após ajuste para potenciais variáveis confundidoras. O cálculo da razão de risco ajustado foi realizado através do software SAS versão 9.1.3 e através da regressão logística multivariada de COX.

\section{RESULTADOS}

No período do estudo, ocorreram 5092 partos, sendo 957 RNs com peso $\leq 2500 \mathrm{~g}$, dos quais 195 gestantes e seus RNs apresentaram os critérios de inclusão para o estudo.

A população de gestantes do estudo apresentou idade média de $22.8 \pm 6.6$ anos (variando de 12 a 43 anos). O número médio de gestações foi de $2.0 \pm 1.6$ gestações (variando de uma a 12), sendo o número médio de partos de $0.7 \pm 1.3$ (variando de zero a oito) e o número de abortos de $0.3 \pm 0.7$ (variando de zero a quatro). A idade gestacional (IG) no momento do internamento, que apresentou média de $32.6 \pm 3.1$ semanas (variando de 24 a 36 semanas e seis dias), foi subdividida em três grupos: $15(7.7 \%)$ tiveram IG $<28$ semanas, 76 (38.9\%) entre 28 e 33 semanas e seis dias e $75(38.5 \%)$ entre 34 e 36 semanas e seis dias. Das 195 pacientes admitidas, $159(81.5 \%)$ apresentaram TPP ativo espontâneo, enquanto 36 (18.5\%) tiveram o diagnóstico de pródromo de TPP e 84 (43.1\%) gestantes apresentavam bolsa rota. Das que se encontravam em pródromo, 15 (41.6\%) foram submetidas à tocólise e em 21 (58.3\%) foi administrado corticoide. O parto vaginal ocorreu em 145 (74.4\%) gestantes e a cesárea em 50 (25.6\%). As principais indicações de cesárea foram: apresentações anômalas (36\%), bolsa rota (22\%), cesárea anterior e bolsa rota (16\%). As demais causas juntas corresponderam a $26 \%$ do total. Não ocorreu nenhum parto fórcipe.

22 RNs (11.3\%) estavam em apresentações anômalas, sendo $18(81.9 \%)$ pélvicas e $4(18.1 \%)$ córmicas, todos nasceram de parto abdominal. Os escores de Apgar ao $1^{\circ}$ e $5^{\circ}$ minutos foram $<7$ em $24.9 \%$ e $6 \%$ dos casos, respectivamente. Os RNs pesaram, em média, $1873 \pm 479 \mathrm{~g}$ (variando de $565 \mathrm{~g}$ a $2885 \mathrm{~g}$ ). Foram subdivididos em quatro grupos: $1^{\circ}$ ) RNs menores que $1000 \mathrm{~g}(\mathrm{n}=15$, correspondendo a $\left.7.7 \%) ; 2^{\circ}\right)$ entre $1000 \mathrm{~g}$ e $1499 \mathrm{~g}(\mathrm{n}=25$, correspondendo a $\left.12.8 \%) ; 3^{\circ}\right)$ entre $1500 \mathrm{~g}$ e $2499 \mathrm{~g}(\mathrm{n}=153$, correspondendo a $78.4 \%)$ e $\left.4^{\circ}\right)$ maiores que $2500 \mathrm{~g}(\mathrm{n}=2$, correspondendo a $1.1 \%)$. Em relação ao Capurro, 109 (55.9\%) RNs foram classificados com IG entre 34 e 36 semanas e seis dias; 76 (39\%) entre 28 e 33 semanas e seis dias e 10 (5.1\%) com menos de 28 semanas. Quanto à adequação do peso à IG, 145 (76.7\%) RNs foram classificados como adequados para a IG; 38 (20.1\%) como pequenos para a IG e seis $(3.2 \%)$ como grandes para a IG. Em seis casos não foi informada a adequação do peso devido ao peso e IG serem inferiores ao mínimo necessário para se fazer o cálculo. 41 (21\%) RNs fizeram uso de surfactante e 177 (90.8\%) dos RNs necessitaram de algum tipo de ventilação. Nove (4.6\%) RNs apresentaram tocotraumatismos. 121 (62.1\%) necessitaram de UTI neonatal. Não ocorreu nenhum óbito fetal na amostra analisada. Ocorreram 17 óbitos neonatais $(8.7 \%)$, sendo 12 precoces $(6.1 \%)$ e cinco tardios $(2.6 \%)$, cujas causas foram sepse $(52.9 \%)$, insuficiência cárdio-respiratória $(35.3 \%)$ e falência de múltiplos órgãos (11.8\%). Nenhum desses resultados apresentou diferenças estatisticamente significativas quando se compararam parto vaginal e cesárea, após avaliação pelo teste de Mann-Whitney e análise multivariada pela regressão logística de COX.

Entre os grupos submetidos ao parto vaginal e à cesárea, não foram observadas diferenças significativas em relação à idade 
materna, número de gestações, paridade, número de abortos e idade gestacional média.

Aumentaram, significativamente, o risco de cesárea o pródromo de TPP (RR 6.10, IC 95\% - 3.97 a 9.37); bolsa rota (RR 1.64, IC 95\% - 1.01 a 2.64); uso de tocolíticos (RR 1.95, IC $95 \%$ - 1.07 a 3.56) e corticoides (RR 1.82, IC 95\% - 1.04 a 3.1). O TPP ativo diminuiu, significativamente, esse risco (RR 0.16, IC 95\% - 0.11 a 0.25), (Tabela 1) e (Figura 1), dados estes em acordo com os do estudo de Jonas et al. ${ }^{18}$

A cesárea aumentou significativamente a chance do índice de Apgar ao $5^{\circ}$ minuto ser $\geq 7$ (RR 1.06, IC 95\% - 1.01 a 1.13).
Quanto aos demais resultados perinatais (adequação do peso à IG, uso de surfactante e número de doses, tocotraumatismos, admissão em UTI neonatal, necessidade de ventilação, tempo de internamento em UTI, tempo de internamento total e óbito neonatal), não ocorreram diferenças, estatisticamente significativas, entre os dois grupos (Tabela 2) e (Figura 2).

Quando foi realizada análise multivariada pela regressão logística de COX, ajustada para fatores que poderiam influenciar nos resultados perinatais, não foram observadas diferenças estatisticamente significativas entre os grupos do parto vaginal e da cesárea (Tabela 3) e (Figura 3).

Tabela 1. Características obstétricas na internação segundo a via de parto.

\begin{tabular}{|c|c|c|c|c|c|}
\hline & Parto vaginal & Cesárea & Incidência de cesárea (\%) & RR & IC $(95 \%)$ \\
\hline \multicolumn{6}{|l|}{ Pródr. TPP } \\
\hline Não & 138 & 21 & 13,2 & 1,00 & \\
\hline Sim & 7 & 29 & 80,6 & 6,10 & $(3,97$ a 9,37$)$ \\
\hline \multicolumn{6}{|l|}{ TPP ativo } \\
\hline Não & 7 & 29 & 80,6 & 1,00 & \\
\hline Sim & 138 & 21 & 13,2 & 0,16 & $(0,11$ a 0,25$)$ \\
\hline \multicolumn{6}{|l|}{$\mathrm{BR}(<18 \mathrm{~h})$} \\
\hline Não & 88 & 23 & 20,7 & 1,00 & \\
\hline Sim & 56 & 28 & 33,3 & 1,64 & $(1,01$ a 2,64$)$ \\
\hline \multicolumn{6}{|l|}{ Tocólise } \\
\hline Não & 137 & 43 & 23,9 & 1,00 & \\
\hline Sim & 8 & 7 & 46,7 & 1,95 & $(1,07$ a 3,56$)$ \\
\hline \multicolumn{6}{|l|}{ Corticoide } \\
\hline Não & 133 & 41 & 23,6 & 1,00 & \\
\hline Sim & 12 & 9 & 42,9 & 1,82 & $(1,04$ a 3,19$)$ \\
\hline \multicolumn{6}{|l|}{ IG } \\
\hline S/inf & 20 & 9 & & & \\
\hline$<28$ s & 14 & 1 & 6,7 & 1,00 & \\
\hline $28-34 \mathrm{~s}$ e $6 \mathrm{~d}$ & 61 & 15 & 19,7 & 2,96 & $(0,42$ a 20,75$)$ \\
\hline $34-36 s$ e $6 d$ & 50 & 25 & 33,3 & 5,00 & $(0,73$ a 34,12$)$ \\
\hline
\end{tabular}

RR = razão de risco; IC 95\% = intervalo de confiança (95\%), Pródr. = pródromo; TPP = trabalho de parto prematuro; $\mathrm{BR}=$ bolsa rota; $\mathrm{IG}=$ idade gestacional; $\mathrm{S} / \mathrm{inf}=$ sem informação; $\mathrm{s}=$ semanas; $\mathrm{d}=$ dias.

Figura 1. Razão de incidência da cesárea em relação às características obstétricas no internamento.

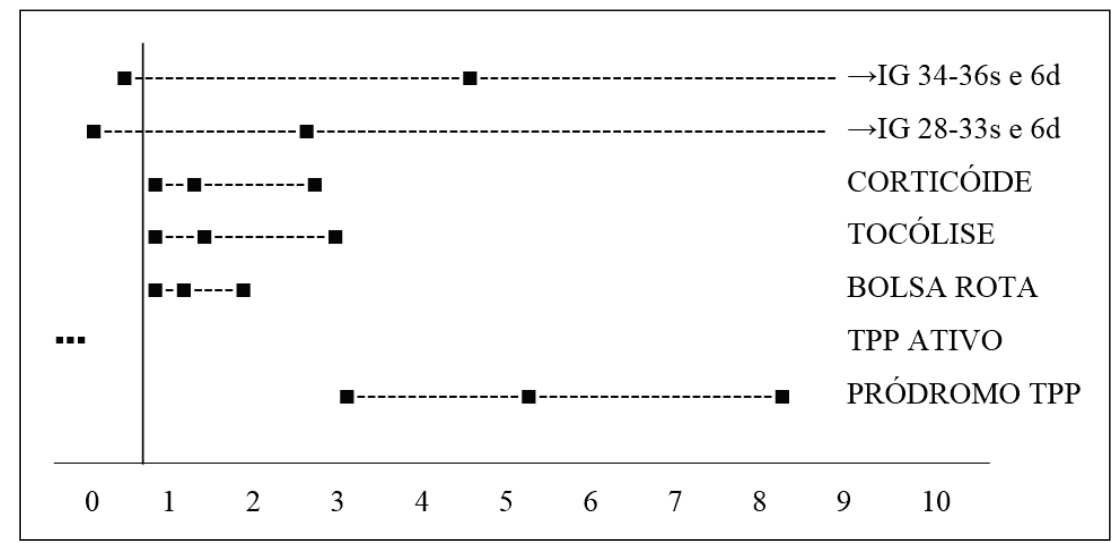


Tabela 2. Resultados perinatais segundo a via de parto.

\begin{tabular}{|c|c|c|c|c|c|c|}
\hline & Parto vaginal & $\begin{array}{c}\text { Incidência } \\
(\%)\end{array}$ & Cesárea & $\begin{array}{c}\text { Incidência } \\
(\%)\end{array}$ & $\mathrm{RR}$ & IC (95\%) \\
\hline \multicolumn{7}{|l|}{ Apgar $1^{\circ} \min$} \\
\hline S/inf & 2 & & & & & \\
\hline$<7$ & 31 & 21,7 & 17 & 34,0 & 1,00 & \\
\hline$\geq 7$ & 112 & 78,3 & 33 & 66,0 & 0,84 & $(0,68$ a 1,05$)$ \\
\hline \multicolumn{7}{|l|}{ Apgar $5^{\circ} \min$} \\
\hline S/inf & 13 & & & & & \\
\hline$<7$ & 10 & 7,6 & 1 & 2,0 & 1,00 & \\
\hline$\geq 7$ & 122 & 92,4 & 49 & 98,0 & 1,06 & $(1,01$ a 1,13$)$ \\
\hline \multicolumn{7}{|l|}{ Peso RN } \\
\hline$<1000 \mathrm{~g}$ & 13 & 9,0 & 2 & 4,0 & 1,00 & \\
\hline $1000-1499 \mathrm{~g}$ & 23 & 15,9 & 2 & 4,0 & 0,78 & $(0,28$ a 2,15$)$ \\
\hline $1500-2499 \mathrm{~g}$ & 107 & 73,8 & 46 & 92,0 & 1,07 & $(0,99$ a 1,17$)$ \\
\hline$\geq 2500 \mathrm{~g}$ & 2 & 1,3 & 0 & 0,0 & não & estimável \\
\hline \multicolumn{7}{|l|}{ Adq. peso/IG } \\
\hline S/inf & 7 & & 1 & & & \\
\hline AIG & 105 & 75,0 & 40 & 81,6 & 1,00 & \\
\hline PIG & 32 & 22,9 & 6 & 12,3 & 0,56 & $(0,25$ a 1,25$)$ \\
\hline GIG & 3 & 2,1 & 3 & 6,1 & 2,51 & $(0,53$ a 11,96$)$ \\
\hline \multicolumn{7}{|l|}{ Capurro } \\
\hline$<28 \mathrm{~s}$ & 8 & 5,6 & 2 & 4,0 & 1,00 & \\
\hline $28-33 s$ e $6 d$ & 63 & 43,4 & 13 & 26,0 & 0,98 & $(0,79$ a 1,21$)$ \\
\hline $34-36 s$ e $6 d$ & 74 & 51,0 & 35 & 70,0 & 1,05 & $(0,94$ a 1,16$)$ \\
\hline \multicolumn{7}{|l|}{ Surfactante } \\
\hline Não & 112 & 77,2 & 42 & 84,0 & 1,00 & \\
\hline Sim & 33 & 22,8 & 8 & 16,0 & 0,70 & $(0,35$ a 1,42$)$ \\
\hline \multicolumn{7}{|c|}{ Tocotraumatismo } \\
\hline Não & 140 & 96,6 & 46 & 92,0 & 1,00 & \\
\hline Sim & 5 & 3,4 & 4 & 8,0 & 2,32 & $(0,65$ a 8,30$)$ \\
\hline \multicolumn{7}{|l|}{ UTI neonatal } \\
\hline Não & 56 & 38,6 & 18 & 36,0 & 1,00 & \\
\hline Sim & 89 & 61,4 & 32 & 64,0 & 1,04 & $(0,82$ a 1,33$)$ \\
\hline \multicolumn{7}{|l|}{ Ventilação } \\
\hline Não & 14 & 9,7 & 4 & 8,0 & 1,00 & \\
\hline Sim & 131 & 90,3 & 46 & 92,0 & 1,02 & $(0,92$ a 1,12$)$ \\
\hline \multicolumn{7}{|l|}{ Óbito neonatal } \\
\hline Não & 131 & 90,3 & 47 & 94,0 & 1,00 & \\
\hline Sim & 14 & 9,7 & 3 & 6,0 & 0,62 & $(0,19$ a 2,07$)$ \\
\hline
\end{tabular}

$\mathrm{RR}=$ razão de risco; IC 95\% = intervalo de confiança (95\%); S/inf = sem informação; Adq = Adequação; RN = recém-nascido; IG = idade gestacional; $\mathrm{AIG}=$ adequados para idade gestacional; PIG = pequenos para idade gestacional; $\mathrm{GIG}=$ grandes para idade gestacional; $\mathrm{s}=$ semanas; $\mathrm{d}=$ dias; $\mathrm{UTI}=$ unidade de terapia intensiva. 
Figura 2. Razão de incidência dos resultados perinatais em relação à via de parto.

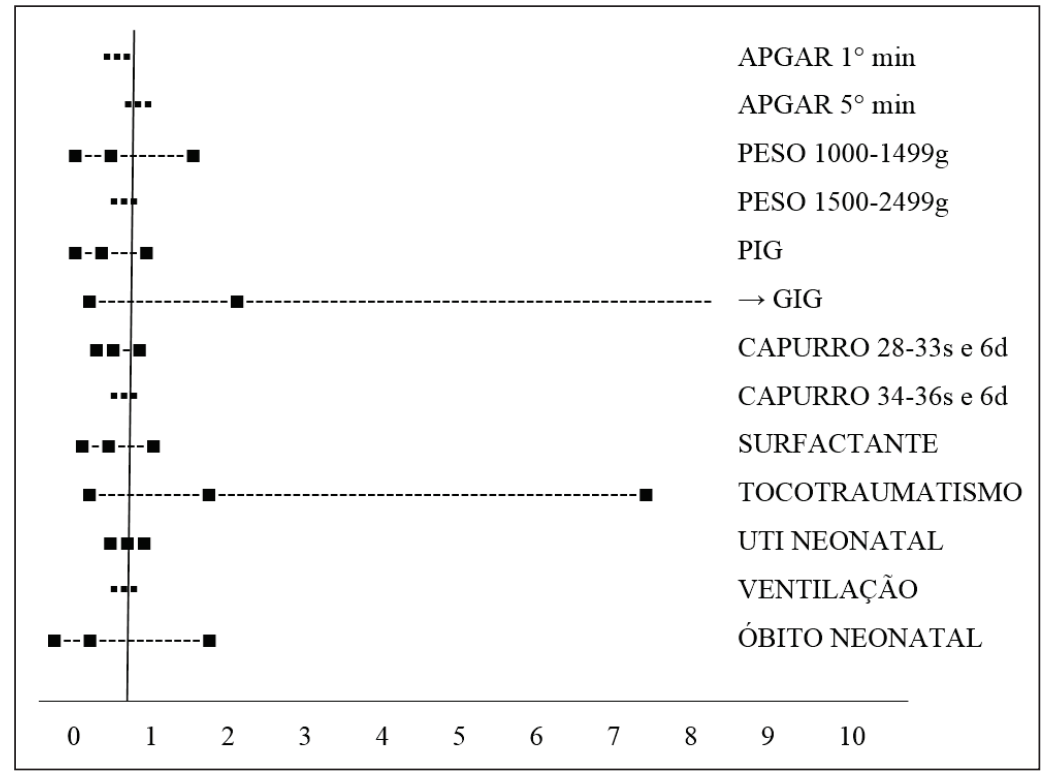

Tabela 3. Influência da via de parto sobre os resultados perinatais após análise multivariada pela regressão logística de COX.

$\begin{array}{lllll}\text { Parto vaginal } & \text { Incidência } & \text { Cesárea } & \text { Incidência } & \text { RR* }\end{array}$

$(\%)$

$(\%)$

\section{Apgar $1^{\circ} \min$}

S/inf

$<7$

$\geq 7$

Apgar $5^{\circ} \mathrm{min}$

S/inf

$<7$

$\geq 7$

Adequação peso/IG

S/inf

AIG

PIG

GIG

Capurro

$<28$ s

$28-33$ s e $6 \mathrm{~d}$

$34-36$ s e $6 \mathrm{~d}$

Surfactante

Não

Sim

Tocotraumatismo

Não 140

Sim

UTI neonatal

$\begin{array}{ll}\text { Não } & 56 \\ \text { Sim } & 89\end{array}$

Continua.

\section{2}

31

13

10

122

105

32

3

8

63

74

112

33

5

56

89
17

33

7,6

92,4

75,0

22,9

2,1

5,5

43,4

51,0

77,2

22,8

96,6

3,4

38,6

61,4

18

32

33

3

49

$$
1
$$

40

34,0

66,0

1,00

0,77

$(0,52$ a 1,22$)$

$\begin{array}{cr}2,0 & 1,00 \\ 98,0 & 1,00\end{array}$

$(0,69$ a 1,47$)$

81,6

1,00

12,2

0,79

$(0,29$ a 2,17$)$

6,1

não

Estimável

$\begin{array}{ccc}4,0 & 1,00 & \\ 26,0 & 0,35 & (0,11 \text { a } 1,15) \\ 70,0 & 1,20 & (0,76 \text { a } 1,90)\end{array}$$$
84,0
$$$$
1,00
$$$$
16,0 \quad 1,66
$$

$8 \quad 16,0 \quad 1,66 \quad(0,69$ a 4,01$)$


Conclusão.

Tabela 3. Influência da via de parto sobre os resultados perinatais após análise multivariada pela regressão logística de COX.

\begin{tabular}{|c|c|c|c|c|c|c|}
\hline & Parto vaginal & $\begin{array}{c}\text { Incidência } \\
(\%)\end{array}$ & Cesárea & $\begin{array}{c}\text { Incidência } \\
(\%)\end{array}$ & $\mathrm{RR}^{*}$ & IC (95\%) \\
\hline \multicolumn{7}{|c|}{ Ventilação mecânica } \\
\hline Não & 14 & 9,7 & 4 & 8,0 & 1,00 & \\
\hline Sim & 131 & 90,3 & 46 & 92,0 & 1,06 & $(0,73$ a 1,56$)$ \\
\hline \multicolumn{7}{|c|}{ Óbito neonatal } \\
\hline Não & 131 & 90,3 & 47 & 94,0 & 1,00 & \\
\hline Sim & 14 & 9,7 & 3 & 6,0 & 3,29 & $(0,78$ a 13,98$)$ \\
\hline
\end{tabular}

(*) Avaliação ajustada através de regressão logística de COX. RR = razão de risco; IC = intervalo de confiança (95\%); S/inf= sem informação; IG = idade gestacional; $\mathrm{AIG}$ = adequados para idade gestacional; PIG = pequenos para idade gestacional; $\mathrm{GIG}=$ grandes para idade gestacional; $\mathrm{s}=$ semanas; $\mathrm{d}=$ dias; $\mathrm{UTI}=$ unidade de terapia intensiva .

Figura 3. Razão de incidência dos resultados perinatais segundo parto após regressão logística de COX.

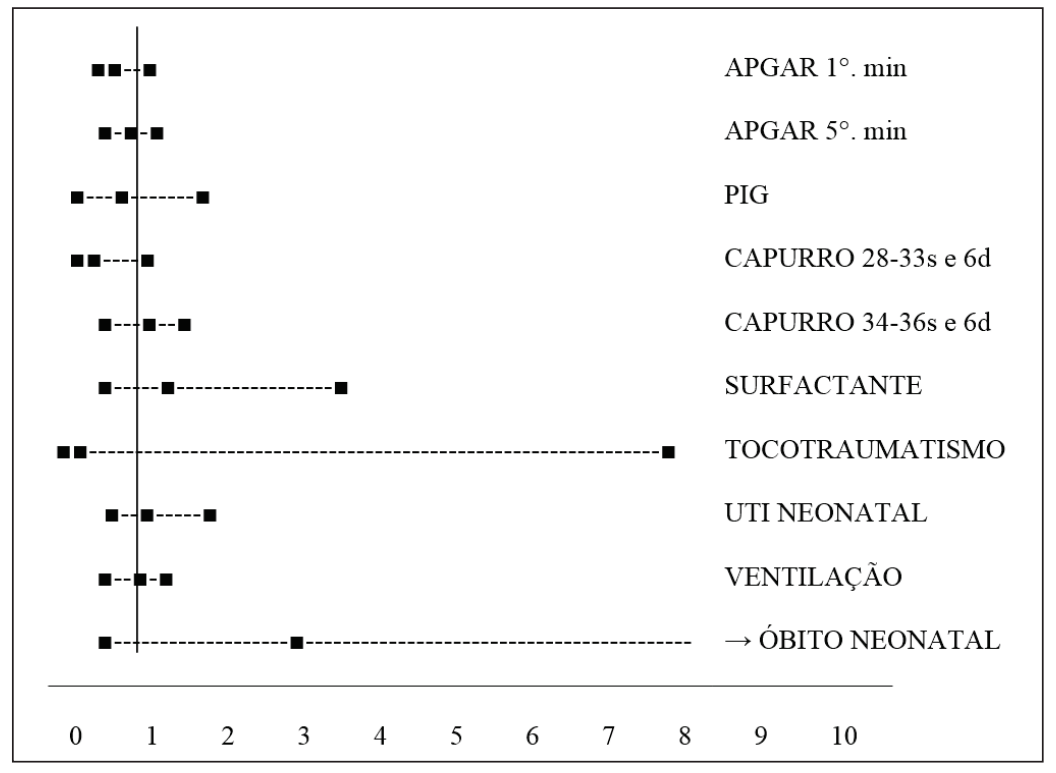

\section{DISCUSSÃO}

O aumento nas intervenções médicas na prematuridade, que incluiu avanços nos cuidados obstétricos e neonatais, contribuiu para o declínio dos resultados perinatais adversos. No entanto, houve aumento na incidência de PP, que continuam sendo a principal causa de morbimortalidade perinatal. ${ }^{19}$ No presente estudo, o índice de RNs pesando $\leq 2500 \mathrm{~g}$, foi acima dos dados citados na literatura, provavelmente pelo fato da MEAC ser referência no atendimento a gestantes de alto risco de toda região metropolitana de Fortaleza e de várias cidades do interior do estado do Ceará.

Nesta casuística, observou-se que a distribuição da população de acordo com a idade gestacional calculada pelo Capurro somático está parcialmente de acordo com os dados de Goldenberg et al. ${ }^{20,1} \mathrm{~A}$ análise da paridade, IM e IG não mostrou diferenças, estatisticamente significativas, como determinantes da via de parto.
Um estudo já havia demonstrado que a morbidade e mortalidade perinatais eram inversamente proporcionais à $\mathrm{IG}$ e ao peso após o nascimento. ${ }^{21}$

Este estudo foi consistente com avaliação prévia onde RNs prematuros de mulheres que foram submetidas à cesárea tiveram maiores idades gestacionais e pesos, quando comparados com os de parto vaginal. ${ }^{14}$ Quanto aos resultados perinatais, os achados deste estudo não mostraram diferenças estatisticamente significativas entre os vários subgrupos de peso e IG, quando comparado parto vaginal ou cesárea.

Após análise multivariada ajustada para fatores que poderiam influenciar nos resultados, as diferenças nos escores de Apgar não apareceram como estatisticamente significativas. Estes dados estão de acordo com os da avaliação de Jonas et al., que mostrou após análise multivariada ajustada, não haver associação estatisticamente significativa entre escore de Apgar < 4 e a via de parto (odds ratio (OR) 0.79 , IC 95\% 0.41 a 1.49$){ }^{22}$ 
O principal achado deste estudo é que a via de parto não influenciou a morbimortalidade perinatal, quando fatores de risco foram controlados. Porém, diante das dificuldades relatadas, suas implicações práticas são limitadas, principalmente em relação às apresentações anômalas, onde não ocorreram partos vaginais e aos grupos de menor IG e menor peso, devido ao pequeno número de recém-nascidos avaliados.

\section{REFERÊNCIAS}

1. Goldenberg RL, Culhane JF, Iams JD, Romero R. Epidemiolog and causes of preterm birth. Lancet. 2008;371(9606):75-84.

2. Shariati KM, Karimi Z, Rezaienejad M, Basiri A, Torkestani F, Saleh Gargari S, Perinatal complications associated with preterm deliveries at 24 to 33 weeks and 6 days gestation (2011-2012): a hospital-based retrospective study. Iran J Reprod Med. 2015;13(11):697-702.

3. Gonik B, Creasy RK. Preterm labor: its diagnosis and management. Am J Obstet Gynecol. 1986;154(1):3-8.

4. ACOG technical bulletin. Preterm labor. Number 206--June 1995 (Replaces No. 133, October 1989). Int J Gynaecol Obstet. 1995;50(3):303-13.

5. Roberts D, Dalziel S. Antenatal corticosteroids for accelerating fetal lung maturation for women at risk of preterm birth. Cochrane Database Syst Rev. 2006;(3):CD004454.

6. Wapner RJ, Sorokin Y, Thom EA, Johnson F, Dudley DJ, Spong CY, et al. Single versus weekly courses of antenatal corticosteroids: evaluation of safety and efficacy. Am J Obstet Gynecol. 2006;195(3):633-42.

7. Royal College of obstetrician \& gynaecologists. Antenatal Corticosteroids to Reduce Neonatal Morbidity (Green-top Guideline No. 7). London; 2010. [acesso em 10 nov. 2017]. Disponível em: https://www.rcog.org.uk/en/guidelines-research-services/guidelines/ $\operatorname{gtg} 7 /$

8. Verani JR, McGee L, Schrag SJ, Division of Bacterial Diseases, National Center for Immunization and Respiratory Diseases. Prevention of Perinatal Group B Streptococcal Disease: Revised Guidelines from CDC, 2010: Recommendations and Reports [Internet]. Atlanta (GA): Centers for Disease Control and Prevention; 2010. [acesso em 03 mai 2016]. Disponível em: https://www.cdc. gov/mmwr/preview/mmwrhtml/rr5910a1.htm

9. Drife J. Mode of delivery in the early preterm infants ( $<28$ weeks). BJOG. 2006;113(Suppl 3):81-5.

10. Bergenhenegouwen LA, Meertens LJ, Schaaf J, Nijhuis JG, Mol BW, Kok M, et al. Vaginal delivery versus caesarean section in preterm breech delivery: a systematic review. Eur J Obstet Gynecol Reprod Biol. 2014;172:1-6.

11. Deutsch A, Salihu HM, Lynch O, Marty PJ, Belogolovkin V. Cesarean delivery versusvaginal delivery: impact on survival and
A falta de evidências, atualmente, em favor da cesárea também não nos permite concluir que existam claras evidências favoráveis ao parto por via vaginal. Para elucidar o verdadeiro impacto da via de parto nos recém-nascidos prematuros, estudos prospectivos e randomizados devem ser realizados.

Os dados deste trabalho são consistentes com a maioria dos encontrados na literatura, no sentido de que a via de parto não influencia os resultados perinatais.

morbidity for the breech fetus at the threshold of viability. J Matern Fetal Neonatal Med. 2011;24(5):713-7.

12. Grant A, Glazener CM. Elective cesarean section versus expectant management for delivery of the small baby. Cochrane Database Syst Rev. 2001;(2):CD000078.

13. Malloy MH. Impact of cesarean section on neonatal mortality rates among very preterm infants in the United States, 2000-2003. Pediatrics. 2008;122(2):285-92.

14. Malloy MH, Doshi S. Cesarean section and the outcome of very preterm and very low birth weight infants. Clin Perinatol. 2008;35(2):421-35.

15. Wylie BJ, Davidson LL, Batra M, Reed SD. Method of delivery and neonatal outcome in very low-birthweight vertex-presenting fetuses. Am J Obstet Gynecol. 2008;198(6):640.e1-640.e7.

16. Malloy MH. Impact of cesarean section on intermediate and late preterm births: United States, 2000-2003. Birth. 2009;36(1):26-33.

17. Furukawa S, Sameshima H, Ikenoue T. The impact of cesarean section on neonatal outcome of infants born at 23 weeks of gestation. Early Hum Dev. 2014;90(3):113-8.

18. Jonas HA, Khalid N, Schwartz SM. The relationship between cesarean section and neonatal mortality in very-low-birthweight infants born in Washington State, USA. Paediatr Perinat Epidemiol. 1999;13(2):170-89.

19. Colvin M, McGuire W, Fowlie PW. Neurodevelopmental outcomes after preterm birth. BMJ. 2004;329(7479):1390-3.

20. Manuck TA, Herrera CA, Korgenski EK, Jackson M, Stoddard GJ, Porter TF, et al. Tocolysis for Women With Early Spontaneous Preterm Labor and Advanced Cervical Dilation. Obstet Gynecol. 2015;126(5):954-61.

21. Joseph KS, Kramer MS, Allen AC, Cyr M, Fair M, Ohlsson A, et al. Gestational age and birthweight-specific declines in infant mortality in Canada, 1985-94. Paediatr Perinat Epidemiol. 2000;14(4):332-9.

22. Jonas HA, Lumley JM. The effect of mode of delivery on neonatal mortality in very low birth weight infants born in Victoria, Australia: Cesarean section is associated with increased survival in breech-presenting, but not vertex-presenting, infants. Paediatr Perinat Epidemiol. 1997;11(2):181-99.

\section{Como citar:}

Mota TG, Alencar CA Junior, Ribeiro GG, Feitosa IS, Feitosa FE. Influência da via de parto sobre os resultados perinatais de mulheres que tiveram parto prematuro. Rev Med UFC. 2018 jan-mar;58(1):37-44. 